Deniz Özkut

\title{
Preserving and documenting the Cultural Heritage
}

Asst.Prof.Deniz Özkut, architect, Assistant Professor

Anadolu University, Iki Eylul Campus,

Faculty of Engineering and Architecture,

Department of Architecture,

Program of Restoration,

Eskiehir, Turkiye

Turkey

dozkut@anadolu.edu.tr; dozkut@gmail.com 


\section{Deniz Özkut}

\section{Preserving and documenting the Cultural Heritage}

The phenomenon of preservation may be described as a physical process that depends on conceptual facts. When observed from a conceptual point of view, 'values' cause the divergences in the preservation process, which could be named as the pre-requisites of the construction. Values carry emotional and physical points of view. The emotional context, of course, depends on recognizing and remembering while physical context depends on direction of research. They are indicators of cultural characteristics and historical identity. 'Cultural values'1, moreover, explains the meanings attributed to the cultural property, which meanings will be preserved, and the reasons for their preservation. On behalf of this context, the preservation process may be defined as the preservation of the cultural heritage within an effective system. This effective system is aimed at attaining the total quality as a result of a synthesis of the technology, technique, and material originally deployed with those of the present. Besides, one of the most important input in the preservation process is the priorities of the intervention to be held, as the latter will determine the decisions and types of intervention during the implementation phase of the preservation project. As a significant paradox, the most important parameter that shapes both a preservation project and its process appears to be the risks that consist of indefinite input preventing the project from a proper definition of its context. Since all physical problems and the social status of the cultural property to be preserved have direct impact upon the design process of the preservation project, these priorities and the risks should be clarified in the course of pre-assessment phase at the beginning of the preservation process.

The essential aim of this pre-assessment phase is to differentiate the indefinite initial information about data relevant to the origin of the risks in order to prevent the intervention priorities from causing any refractory consequences from occurring at the end of the project process. The more the risks related to the building to be preserved are identified, the more the attitude to be adopted for that cultural property in question will become definite. In order to achieve a sound decision for preservation, it is essential to obtain comprehensive, correct, utilizable, and applicable information about the context in the pre-assessment phase.

Architectural documentation is not only the essential initial work of the pre-assessment phase but also one of the medium of representation with respect to "digital integrated with analogue" approach. Prior to this pre-assessment phase, it is concerned with the investigation, analysis, definition, understanding, and solution of the problems of the existing construction. Subse-

97 - ARCC Journal / Volume 5 Issue $2 \quad-97$ 
quently, there resides the requisite to the implementation and design phases of the preservation project. This data, then, will be transformed into detailed and accurate information through the proper process. In regard to this point of view, there reside two parallel works that are juxtaposed in the particular preservation project: restitution proposal along with implementation project. To have a sound restitution, there should be rendered a comprehensive and accurate architectural documentation work indeed. With respect to this requisite, the concern in this paper is the representation of the cultural property, due to protect its cultural, physical, emotional or economical values. Additionally, the utilisation of the advanced technologies for architectural documentation will be considered from the architectural point of view.

As a key concept, documentation reveals as one of the forms of representation. Since, documentation work constitutes an architectural basis for a sound restitution by means of the data and information that are derived in the course of documentation phase. However, this documentation process, itself, may especially emerge as bearing risk that consists of indefinite input preventing the project from a proper context definition, depending upon the selected technology and the method. On behalf of the preservation process, digital medium provides flexibility and relative speed in every phase of conservation. Digital medium reduces the extent of documentation process while increasing the precision of the documentation. Recently, reconstruction of a cultural property via digital technology emerges as being proper to experience the spatial characteristics of that cultural property. Therefore, an archaeological case is determined to demonstrate the relation of "cultural property and digital medium" since it includes a long-term decisive and comprehensive solution: an ancient lighthouse construction, which is the unique standing construction attaining from the ancient period in Patara.

On behalf of Patara case, the primary risk is the numerous numbers of unidentified data in the beginning of the process. In addition, the high ratio of risk, depending upon these unidentified data, causes unestimated phases in the entire process of conservation project. In the context of every conservation project, there resides failure not only to restitute the constructions but also to elucidate the conservation priorities related with cost and timing in the beginning unless the assumed risk percentage are kept within the low range.

Though, the end product and the aim of conservation are identified at the beginning theoretically. As far as the process is concerned, the ratio of unexpected and unidentified input ascends seriously. This naturally entails indefiniteness in the conservation process. When it is discussed through the Patara case, unidentified data is the original morphology and typology of the remained construction. Hence, the information about the ancient lighthouse constructions is substantially limited and depends upon hypotheses. On the other hand, there are more than 2000 architectural pieces obtained in the course of excavation. Before elucidation and identification of this risky data, it is unachievable either to constitute a restitution project or to produce the implementation decisions with respect to the conservation process.

98 - ARCC Journal / Volume 5 Issue 2 - 98 


\section{Figure 1}

The Patara lighthouse can be described with two different constructions: the cylindiric upper structure and the podium where the cylindiric upper structure stands.

Figure 2

Measured drawings of the construction in the course of the archaeological excavation.


Therefore, there should inevitably be produced architectural documentation. This documentation process produces enormous amount of data to be archived. This archive work could be achievable by means of a well-organized long-term database. At this moment, the integration of digital medium to the process reveals as practical for collecting, archiving or classifying the entire data in a very limited time period.

The subsequent risk is the challenge between rendering conservation process and protecting process with regard to its complicated topography. The cultural property, Patara lighthouse, used to be covered by sand before 2005. Regrettably, the construction has major physical deteriorations and structural deformations after the excavation was completed. Producing a comprehensive and an accurate conservation project urgently in an extremely concise period should be the key concept. The aim is to render the values of Patara Lighthouse sustainable.

The associated following risk parameter is the duration of the preservation process. The longer continues the conservation project (including documentation, restitution \& restoration projects, and implementation steps), the more will be emerged as deteriorated the cultural property. Furthermore, these traditional methods for the documentation take quite long time to complete 

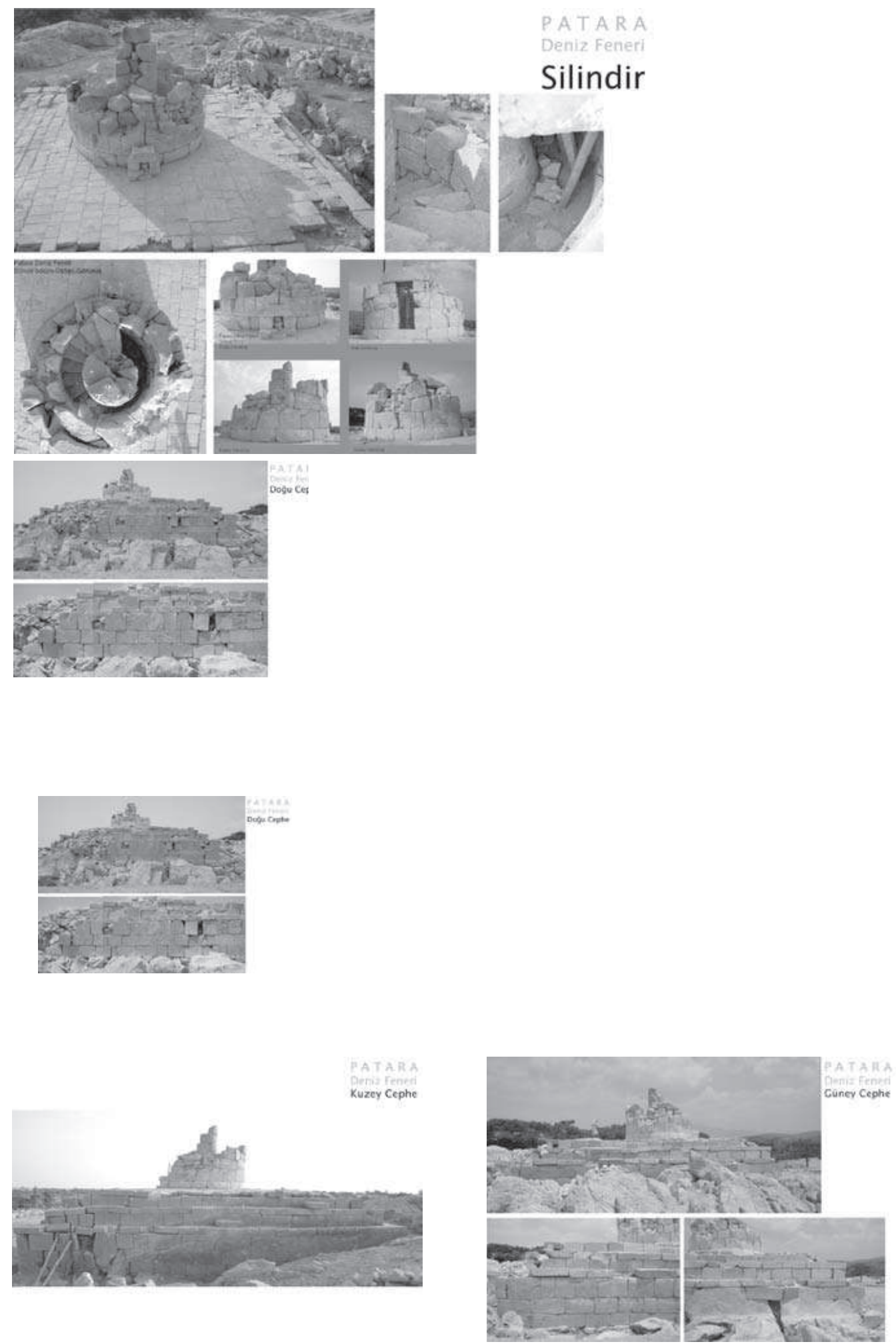

Figure 3

The present situation of the lighthouse, the cultural property, to be documented

100 - ARCC Journal / Volume 5 Issue 2 - 100 

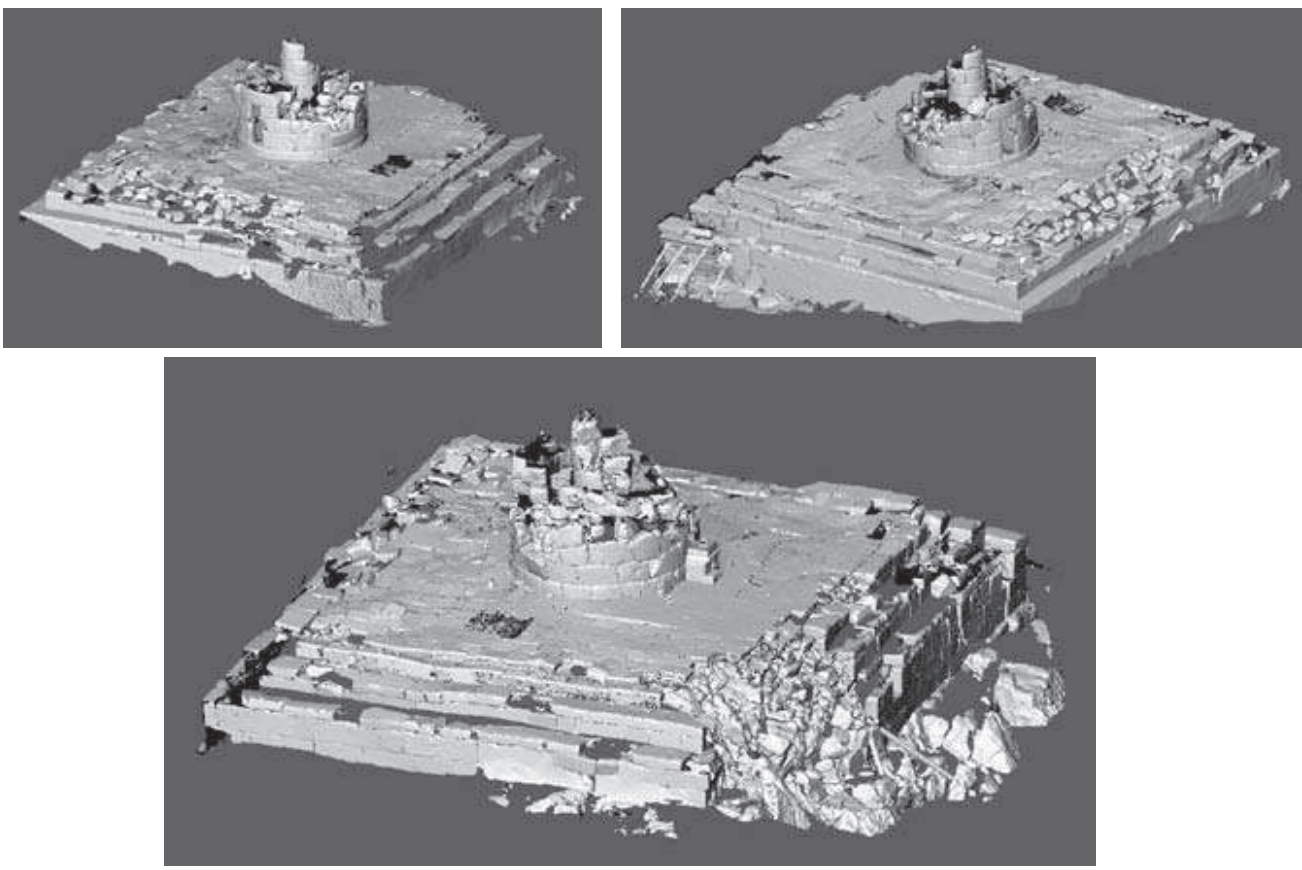

Figure 4

The 3Dpoint cloud data of lighthouse via laser scanner

the qualified work in time despite a standing construction to constitute a preservation strategy about. This time consuming process will challenge the continuation of the cultural property and will be confronted by means of digital technology.

Conventional/traditional/analogue works do not represent such a complicated construction. Digital approaches emerge as the basis for a digital restitution.

\section{Methods Proposed for the Architectural Documentation}

On behalf of digital documentation, it is proposed to produce the accurate and detailed 3D restitution project and 3D architectural inventory. This method decodes the cultural values (uniqueness, historical, documentary, architectural, symbolic, educational and additive values). The latter is achievable to understand the morphology, architectural characteristics, material properties, and the structural techniques and technologies of lighthouse construction. In addition, it is to produce a comprehensive preservation project as well as the priorities and the decisions for restoration. The restitution project could only be relevant by means of a comprehensive, accurate and well organized detailed documentation work. Therefore, 3D measured drawings should be produced in order to describe and identify the construction entirely with respect to the cylindiric light house tower. This situation reveals the significance of the constitution of 3D architectural inventory. This 3D architectural inventory is integrated with the 3D measured drawings or the digital images of the construction in order to produce conservation project.

101 - ARCC Journal / Volume 5 Issue 2 - 101 

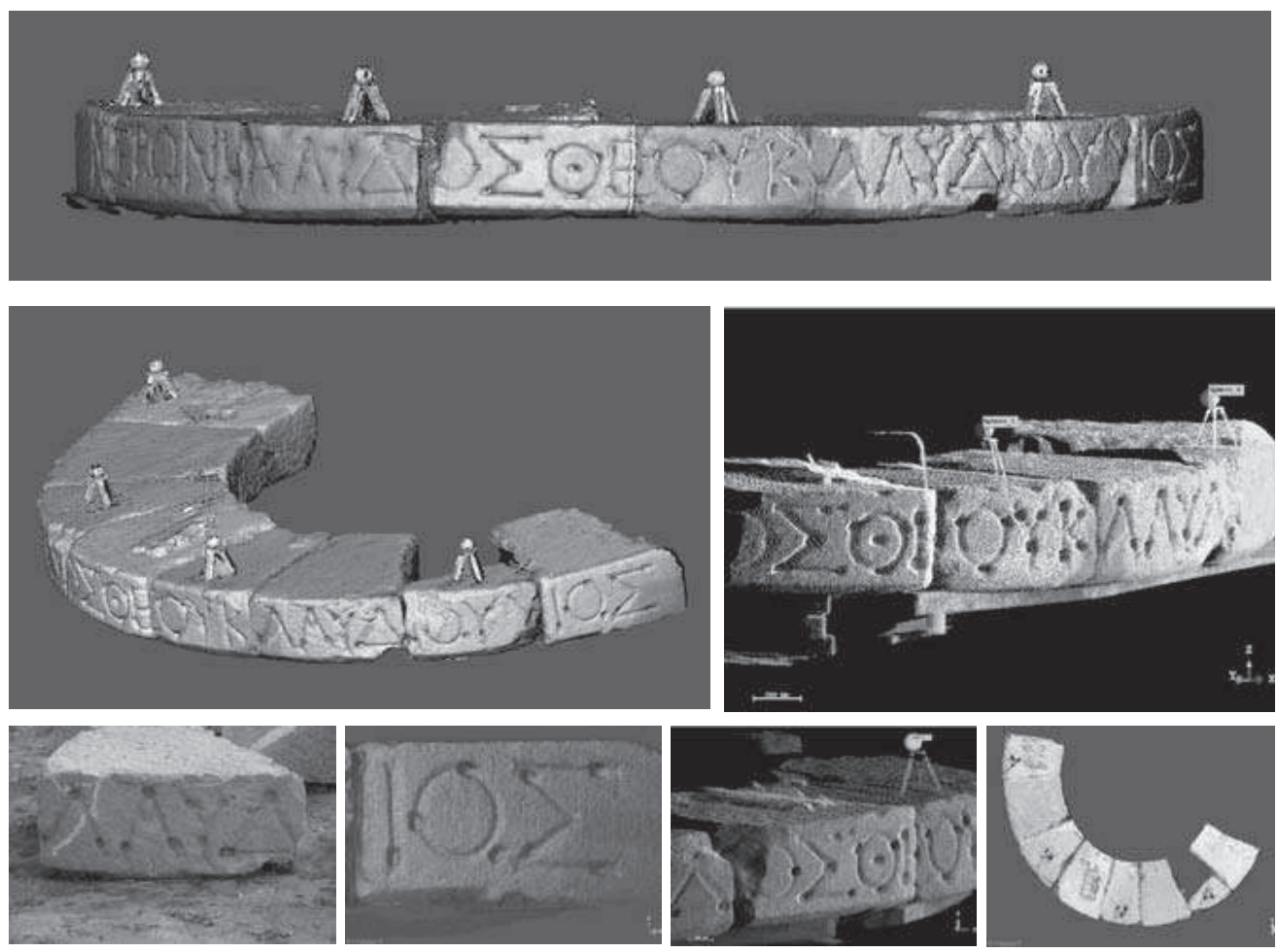

Figure 5

The details of 3D works of lighthouse

Traditional methods cause this pre-assessment process and the constitution of the architectural inventory last in a very long time period besides its difficulties in manipulating the data properly. Advanced technologies are utilized to obtain the accelaration in process, the improvement in information gathering, and the database for architectural inventory.

At the end of the process that combines the archaeology, architecture, preservation and the technology fields, the indefiniteness would be clarified and decoded mostly. With regard to the values and the risks of Patara lighthouse, the point is to render comprehensive digital information in the light of an interdisciplinary work.

\section{CONCLUSION}

The utilization of digital medium is relevant in reducing and identifying the risk parameters in the course of preservation process. At this moment, the most significant and essential object of the entire process needs to be defined as well since the pre-assessment phase revealed as the most significant step and pre-requisite of the entire process. This object must be the cultural property every time. By no means, should not the focus be changed in the course of documentation process. If the focal point shifts to the advantages of these technologies, the cultural property means losing its priority. 
Conservation must preserve and, if possible, enhance the messages and values of cultural property. These values help systematically to set overall priorities in deciding proposed interventions, as well as to establish the extent and nature of individual treatment. This paper has involved in the preservation theories, preservation project, and advanced documentation technologies, which are definitely to be integrated in order not to underestimate the main goal and to discover with a number of issues, as an architect, as

- values to be preserved and propounded

- Reducing the controllable risks.

- the accurate techniques to be chosen and integrated,

- Velocity in accordance with either implementation/intervention or restitution projects.

- The significance of time saving techniques

As a very final word, every preservation project is a unique process to be aware of the context of the cultural property. The aim of achieving the sound preservation on behalf of the pre-requisites is unattainable unless the integrated architectural documentation process is constituted. In conclusion, understanding and representing a cultural heritage becomes significant paradigm in protecting the cultural heritage. Sustainability and continuity of the values should always stand in front of that representation.

\section{Notes}

1 Cultural Values are Continuity value; Uniqueness value; Symbolic value; Impression value, combination of cultural properties and the other values, Architectural value, combination of architectural, morphological, and typographical characteristics of the cultural identity; Historical value, as a result of the production of all cultural items in time and at specific periods; Aging value, related with historical value as well; Documentary value, the reflections of those societies and mode of life on that property; Educational and Documentary values, the need to preserve historical identity; Originality and Uniqueness values, strengthening the documentary, educational, spiritual, and the continuity values.

\section{References}

Ayako,F.,Yuuichi,S., GIS for Cultural Heritage Management in Petra,L.A.Pierce, 2005;A Web-Based GIS System to Manage and Understand Cultural Heritage and Settings. 2005.

Czajkowski, K., Gladki, M., Application of Digital Land-Based Photogrammetry in Architectural and Archaeological Recording. 2005.

Demas,M. Planning For Conservation and Management of Archaeological Sites A Values-Based Approach. 2002. Digital Heritage: Applying Digital Imaging to Cultural Heritage. Ed. L.MacDonald, 2006.

Egels,Y. Digital Photogrammetry, 2001

Jigyasu, R. Towards Developing Methodology for Integrated Risk-Management of Cultural Heritage Sites and Their Settings. 2005.

Özkut, D. "Reducing Risk in 'Preservation Project Management': Re-definition of the 'Pre-assessment Phase'”, PhD, IYTE, 2004.

World Heritage Site Management. UNESCO World Heritage Center, 2003;

103 - ARCC Journal / Volume 5 Issue 2 - 103 